\title{
Análisis de tendencias climáticas con RClimdex en el departamento de Caldas, Colombia
}

\author{
Analysis of climatic trends with RClimdex in Caldas, Colombia. \\ O. L. Ocampo-López iD ; J.J. Vélez-Upegui iD ; T. Forero-Hernández iD ; J.P. Marín-Salazar iD \\ DOI: https://doi.org/10.22517/23447214.22771 \\ Artículo de investigación científica y tecnológica
}

\begin{abstract}
Resumen - La presente investigación buscó establecer la existencia de tendencias en los índices extremos climáticos para el departamento de Caldas. Los resultados se obtuvieron a partir del análisis de series históricas con un registro superior a 30 años, 55 en el caso del estudio de las variables asociadas a precipitación y 10 en el caso de la variable temperatura. El análisis se realizó mediante la herramienta RClimdex, con la cual se identificó la presencia de tendencias crecientes y decrecientes, en aquellas estaciones que presentaron diferencias estadísticamente significativas ( $P$ Values $<0,10$ ) en los indicadores climáticos evaluados. Este análisis de tendencias de variables climatológicas permite asegurar con un nivel de confianza del $90 \%$ que en el departamento podría presentarse un aumento, tanto en la cantidad precipitación como en la duración de los periodos húmedos, pero se concluye que es necesario el fortalecimiento de la red de estaciones en el territorio, con el fin de generar análisis de mayor cobertura, dado que es evidente la ausencia de series históricas en las subregiones Magdalena Caldense, Occidente Próspero y Alto Occidente.
\end{abstract}

Palabras clave- Clima, Eventos extremos, Lluvia, Índices climáticos, Temperatura.

Abstract - The present research sought to establish the existence of trends in the indices of climate extremes for the department of Caldas. The results were obtained from the analysis of historical series with a record superior to 30 years, 55 in the case of the study of the variables associated to precipitation and 10 in the case of the temperature variable. The analysis was performed using the RClimdex tool, which identified the presence of increasing and decreasing trends, in those stations that presented statistically significant differences $(P$ Values $<0.10)$ in the climatic indicators evaluated. This trend analysis of climatological variables allows to ensure with a level of confidence of $90 \%$ that in the department there could be an increase, both in the amount of precipitation and in the duration of the wet periods, but it is concluded that the strengthening of the network of stations in the territory in order to generate analysis of greater coverage, given that the absence of historical series in Magdalena Caldense, Occidente Prospero and Alto Occidente subregions is evident.
Index Terms - Climate, Extreme Events, Rain, Indices, Temperature.

\section{INTRODUCCIÓN}

- $\mathrm{L}$ análisis de tendencias climáticas basado en el tratamiento de series históricas del territorio se ha constituido como una herramienta fundamental en el estudio de la climatología característica de una región, lo cual permite identificar patrones de comportamiento asociados a fenómenos como la variabilidad climática, y en el contexto actual, variaciones producidas por efecto del cambio climático.

La interpretación de los resultados obtenidos mediante estudios de este tipo se vincula con la definición de amenazas climáticas $\mathrm{y}$, por lo tanto, con la formulación de planes y programas para la gestión adecuada del recurso hídrico, el riesgo y el cambio climático, que a su vez articulen los esfuerzos realizados por las autoridades territoriales y los representantes de los diferentes sectores económicos, los cuales propenden por el desarrollo de un territorio climáticamente resiliente.

Según lo reportado en los informes del IPCC [1], se han presentado cambios en los fenómenos meteorológicos y climáticos de naturaleza extrema, algunos asociados a factores antropogénicos como el aumento de la temperatura, la elevación del nivel del mar y el incremento de las precipitaciones intensas en todo el planeta; éste último, se relaciona con el aumento de caudales extremos $\mathrm{y}$, consecuentemente, con el incremento de los indicadores de amenaza y riesgo de inundaciones en el contexto regional.

En Colombia, aunque gran parte de las variaciones en los regímenes de precipitación se atribuyen a la ocurrencia de fenómenos El Niño y La Niña [2], [3], se estima que los eventos extremos serán cada vez más recurrentes por la afectación del cambio climático [1].

Investigadores como Pabón [4], [5], [6], Mesa et al. [7], Pérez et al. [8], Quintana-Gómez [9] , León [10], Pabón et al.

Este manuscrito fue enviado el 30 de agosto de 2019 y aceptado 23 de

J.J. Vélez está vinculado al Departamento de Ingeniería Civil de la Universidad

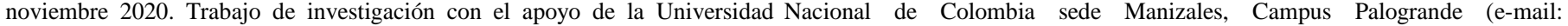
Autónoma de Manizales, la Universidad Nacional de Colombia sede Manizales jjvelezu@unal.edu.co).

y la Corporación Autónoma Regional de Caldas (Corpocaldas). A.T. Forero es estudiante de la Maestría en recursos hidráulicos de la

O. Ocampo está vinculada al Departamento de Mecánica y Producción de la Universidad Nacional de Colombia sede Manizales (e-mail: Universidad Autónoma de Manizales, Antigüa estación del Ferrocarril, atforeroh@unal.edu.co).

Manizales. (e-mail: olocampo@autonoma.edu.co).

J.P. Marín es graduada de la Maestría en recursos hidráulicos de la Universidad Nacional de Colombia sede Manizales (email: jepmarinsa@unal.edu.co). 
[11] , Poveda et al. [12] , Acevedo [13] y Poveda [14] analizaron el fenómeno de cambio climático en Colombia, evidenciando tendencias a largo plazo en las variables temperatura y precipitación. Los diversos estudios permitieron establecer que la temperatura media en el país está aumentando entre 0,1 y $0,2^{\circ} \mathrm{C}$ por década, con un valor promedio de aumento de $0,13^{\circ} \mathrm{C}$ cada 10 años; en el caso de la variable precipitación, se han presentado reducciones en los valles de los ríos Magdalena y Cauca e incrementos en zona del Piedemonte Llanero y la región Pacífica del país.

Instituciones de carácter nacional como el INVEMAR [15], el IDEAM [16], [17] y el Ministerio de Ambiente y Desarrollo Sostenible, han realizado estudios para evaluar los posibles impactos del cambio climático en el país. Se destacan los estudios realizados por el IDEAM, los cuales han permitido continuamente comprender el comportamiento del clima en el país [18], [19] y la estimación de la oferta hídrica [20], [21]. Estos estudios evidencian que en Colombia está aumentando la variabilidad temporal de las series hidrológicas y ciertas áreas experimentan reducciones en su oferta hidrológica natural; no obstante, se evidencian también tendencias de calentamiento a largo plazo en las series de temperatura máxima [22] y mínima [23]. Las tendencias de calentamiento estudiadas permiten a su vez caracterizar olas de calor y frío en el territorio nacional [24], facilitando la determinación de señales de cambio climático [25].

El análisis de tendencias en series climáticas ha sido estudiado por diferentes autores como Pérez-Rendón, Ramírez-Builes, \& Peña-Quiñones [26], quienes mediante el uso del estadístico de Mann-Kendall, identificaron tendencias en las series anuales de temperatura del aire en la región cafetera colombiana y diversas señales de cambio climático. Por otra parte, Mayorga, Hurtado \& Benavides [25] estudiaron posibles tendencias en el periodo 1971-2010 empleando la herramienta RClimdex y obtuvieron evidencias de un aumento generalizado tanto de las precipitaciones, como de las tormentas y lluvias intensas en el país, exceptuando zonas en San Andrés, Arauca, Casanare y Alto Cauca donde se detectaron tendencias de reducción en la precipitación.

Con el objetivo de medir y analizar constantemente las señales de cambio climático, los diversos estudios del IDEAM [27] caracterizan los indicadores que presentan cambios en el sistema climático nacional, teniendo como resultados promedios nacionales de las tasas lineales de calentamiento: $0,15^{\circ} \mathrm{C}$ para el periodo $1961-2010$ y $0,20^{\circ} \mathrm{C}$ para el periodo 1980-201; por otra parte, se estableció el periodo 2001-2010 como el decenio más caluroso y lluvioso, con una temperatura promedio de $22,36^{\circ} \mathrm{C}$ y una precipitación mensual equivalente a $178,5 \mathrm{~mm}$, siendo el año 2010 el más lluvioso, seguido por el año 2011. En cuanto a la variación del nivel del mar, diversos análisis han estudiado su tasa de incremento, variando en el rango de 3 y $5 \mathrm{~mm}$ al año en la costa Pacífica y entre 1 y $2 \mathrm{~mm}$ en la costa Caribe [28], [29], [30], [15].

Según estadísticas de la Organización Meteorológica Mundial, durante los últimos cincuenta años, los eventos meteorológicos extremos han causado nueve de cada diez desastres en el mundo [31], [32]; particularmente la primera década del siglo XXI se caracterizó por la ocurrencia de fenómenos de precipitación extrema, generadores de inundaciones, desbordamientos y deslizamientos [33], [34].

El IPCC [35] define el evento extremo como aquel fenómeno meteorológico "raro" en términos de su distribución estadística de referencia; presenta una baja frecuencia de ocurrencia en el tiempo, esto quiere decir que, entre evento y evento existen periodos extensos [34]. No obstante, los registros evidencian que, en los últimos años, los fenómenos de esta naturaleza han sido más frecuentes e intensos [36], afectando los ecosistemas, el equilibrio en el medio ambiente, los sectores socioeconómicos y las poblaciones [37], especialmente aquellas con menos recursos, deficiente capacidad adaptativa y resiliencia limitada [38], [39], [34].

El análisis de eventos hidroclimáticos extremos permite mejorar la comprensión de la dinámica del clima local tanto en el tiempo como en el espacio [34], [39] y se constituye como una herramienta indispensable para el análisis hidrológico en el cual, se pueden evidenciar señales de la ocurrencia, intensidad y duración de fenómenos de variabilidad climática y cambio climático [3].

El departamento de Caldas, objeto de estudio del presente artículo, se encuentra ubicado en las cordilleras andinas Central y Occidental, que se caracterizan por poseer un clima húmedo y variaciones marcadas en la temperatura durante el día, influenciada por los gradientes altitudinales [40], [41]. El departamento, a pesar de su pequeña extensión, cuenta con cinco pisos térmicos, desde páramos altos hasta zonas cálidas. La abrupta topografía y la amplia variabilidad espacio temporal [40], [42], [43] de la precipitación en el territorio constituyen los factores más preponderantes en la definición de amenazas por deslizamientos.

\section{Metodología}

El análisis de las variables climáticas fue realizado a partir de las series de registros diarios y horarios de las estaciones meteorológicas y pluviométricas administradas por el IDEAM, la CHEC, el Centro Nacional de Investigaciones del Café (CENICAFE), ISAGEN y la Universidad Nacional de Colombia, Sede Manizales.

Las tendencias de las variables climáticas fueron obtenidas mediante el tratamiento de información histórica de las estaciones de monitoreo climático y meteorológico en el departamento de Caldas; para tal fin, se compilaron los resultados del estudio "Modelación hidrológica y agronómica de los efectos del cambio y la variabilidad climática en la producción cafetera de Caldas" [44]. Los índices climáticos analizados se presentan en la Tabla I.

Las series consideradas poseen una extensión superior a 30 años de registro, teniendo un promedio de registro de 42 años en variables asociadas a la precipitación y de 44 años en el registro de variables asociadas a la temperatura. En el caso de las series de precipitación, se consideraron estaciones con registros desde el año 1949 hasta el año 2015, por otra parte, las series de temperatura registran datos de hasta el año 1951. Las Tablas II y III relacionan las estaciones tanto de precipitación como de temperatura, que presentan evidencia de tendencias en su comportamiento. 
TABLA I

ÍNDICES CLIMÁTICOS EXTREMOS [45]

\begin{tabular}{|c|c|c|c|c|}
\hline Variable & ID & $\begin{array}{c}\text { Nombre del } \\
\text { indicador }\end{array}$ & Definición & $\begin{array}{c}\text { Unida } \\
\mathrm{d}\end{array}$ \\
\hline \multirow[t]{8}{*}{$\begin{array}{c}\text { Precipitaci } \\
\text { ón }\end{array}$} & RX1day & $\begin{array}{l}\text { Cantidad } \\
\text { máxima de } \\
\text { precipitación en } \\
\text { un día }\end{array}$ & $\begin{array}{l}\text { Máximo } \\
\text { mensual de } \\
\text { precipitació } \\
\text { n en un día }\end{array}$ & $\mathrm{mm}$ \\
\hline & RX5day & $\begin{array}{l}\text { Cantidad } \\
\text { máxima de } \\
\text { precipitación en } \\
5 \text { días }\end{array}$ & $\begin{array}{l}\text { Máximo } \\
\text { mensual de } \\
\text { precipitació } \\
\text { n en } 5 \text { días }\end{array}$ & $\mathrm{mm}$ \\
\hline & SDII & $\begin{array}{l}\text { Índice simple } \\
\text { de intensidad } \\
\text { diaria }\end{array}$ & $\begin{array}{l}\text { Precipitaci } \\
\text { ón anual } \\
\text { total } \\
\text { dividida } \\
\text { para el } \\
\text { número de } \\
\text { días } \\
\text { húmedos } \\
\text { en un año }\end{array}$ & $\begin{array}{l}\mathrm{mm} / \mathrm{d} \hat{~} \\
\mathrm{a}\end{array}$ \\
\hline & CDD & $\begin{array}{l}\text { Días secos } \\
\text { consecutivos }\end{array}$ & $\begin{array}{l}\text { Número } \\
\text { máximo de } \\
\text { días } \\
\text { consecutiv } \\
\text { os con } \\
\text { RR<1 mm }\end{array}$ & día \\
\hline & CWD & $\begin{array}{l}\text { Días húmedos } \\
\text { consecutivos }\end{array}$ & $\begin{array}{l}\text { Número } \\
\text { máximo de } \\
\text { días } \\
\text { consecutiv } \\
\text { os con } \\
R R \geq 1 \mathrm{~mm}\end{array}$ & día \\
\hline & R95p & $\begin{array}{l}\text { Días muy } \\
\text { húmedos }\end{array}$ & $\begin{array}{l}\text { Precipitaci } \\
\text { ón anual } \\
\text { total en que } \\
\text { RR }>95 \\
\text { percentil }\end{array}$ & $\mathrm{mm}$ \\
\hline & R99p & $\begin{array}{l}\text { Días } \\
\text { extremadament } \\
\text { e secos }\end{array}$ & $\begin{array}{l}\text { Precipitaci } \\
\text { ón anual } \\
\text { total en que } \\
\text { RR }>99 \\
\text { percentil }\end{array}$ & $\mathrm{mm}$ \\
\hline & $\begin{array}{l}\text { PRCPT } \\
\text { OT }\end{array}$ & $\begin{array}{l}\text { Precipitación } \\
\text { total anual en } \\
\text { los días } \\
\text { húmedos }\end{array}$ & $\begin{array}{l}\text { Precipitaci } \\
\text { ón total } \\
\text { anual en } \\
\text { los días } \\
\text { húmedos }\end{array}$ & $\mathrm{mm}$ \\
\hline \multirow[t]{3}{*}{$\begin{array}{c}\text { Temperatur } \\
\text { a }\end{array}$} & TXx & Max Tmax & $\begin{array}{l}\text { Valor } \\
\text { mensual } \\
\text { máximo de } \\
\text { temperatur } \\
\text { a máxima } \\
\text { diaria }\end{array}$ & ${ }^{\circ} \mathrm{C}$ \\
\hline & $\mathrm{TNx}$ & Max Tmin & $\begin{array}{l}\text { Valor } \\
\text { mensual } \\
\text { máximo de } \\
\text { temperatur } \\
\text { a mínima } \\
\text { diaria }\end{array}$ & ${ }^{\circ} \mathrm{C}$ \\
\hline & $\mathrm{TNn}$ & Min Tmin & $\begin{array}{l}\text { Valor } \\
\text { mensual } \\
\text { mínimo de } \\
\text { temperatur } \\
\text { a mínima } \\
\text { diaria } \\
\end{array}$ & ${ }^{\circ} \mathrm{C}$ \\
\hline
\end{tabular}

Las series fueron validadas, tratadas estadísticamente y analizadas con la aplicación RClimdex, para calcular los índices de extremos climáticos y detectar señales de cambio climático.

Para el análisis correspondiente no se complementaron las series históricas, no obstante, se realizaron las pruebas clásicas de homogeneidad.

Estadísticamente, un resultado es significativo cuando es improbable que haya sido debido al azar, para tal efecto se consideró un nivel de significancia del $90 \%$.

\begin{tabular}{|c|c|c|c|c|c|}
\hline \multicolumn{6}{|c|}{$\begin{array}{l}\text { TABLA II } \\
\text { ESTACIONES DE PRECIPITACIÓN CON EVIDENCIA DE } \\
\text { TENDENCIAS } \\
\end{array}$} \\
\hline \multirow{2}{*}{$\begin{array}{l}\text { Municipio } \\
\text { Aguadas }\end{array}$} & \multirow{2}{*}{$\begin{array}{r}\text { Estación } \\
\text { Aguadas }\end{array}$} & \multirow{2}{*}{$\begin{array}{r}\text { Latitud } \\
5,600\end{array}$} & \multirow{2}{*}{$\begin{array}{c}\text { Longitud } \\
-75,450\end{array}$} & \multicolumn{2}{|c|}{$\begin{array}{c}\text { Periodo de } \\
\text { registro }\end{array}$} \\
\hline & & & & 1970 & 2015 \\
\hline Aguadas & La María & 5,583 & $-75,567$ & 1962 & 2015 \\
\hline Aguadas & La Pintada & 5,733 & $-75,583$ & 1980 & 2015 \\
\hline Aguadas & Guaymaral & 5,650 & $-75,450$ & 1961 & 2014 \\
\hline Pácora & $\begin{array}{l}\text { Pacora Plaza } \\
\text { Feria }\end{array}$ & 5,517 & $-75,450$ & 1970 & 2015 \\
\hline Pácora & La Cascada & 5,467 & $-75,550$ & 1983 & 2014 \\
\hline Pácora & La Linda & 5,550 & $-75,533$ & 1983 & 2014 \\
\hline Salamina & Salamina & 5,383 & $-75,483$ & 1970 & 2015 \\
\hline Salamina & San Félix & 5,367 & $-75,367$ & 1970 & 2015 \\
\hline Samaná & Florencia & 5,517 & $-75,033$ & 1978 & 2014 \\
\hline Samaná & La Palma & 5,517 & $-75,033$ & 1964 & 2014 \\
\hline Norcasia & Norcasia & 5,164 & $-75,517$ & 1979 & 2015 \\
\hline Norcasia & El Vergel & 5,566 & $-74,881$ & 1980 & 2014 \\
\hline Victoria & La Victoria & 5,317 & $-74,900$ & 1974 & 2015 \\
\hline Victoria & La Pastorita & 5,317 & $-74,967$ & 1979 & 2014 \\
\hline Victoria & Cañaveral & 5,321 & $-74,942$ & 2012 & 2014 \\
\hline Manzanares & Manzanares & 5,250 & $-75,133$ & 1972 & 2014 \\
\hline Manzanares & Llanadas & 5,200 & $-75,133$ & 1956 & 1998 \\
\hline Marquetalia & Marquetalia & 5,283 & $-75,050$ & 1963 & 2015 \\
\hline Marquetalia & Santa Helena & 5,318 & $-74,996$ & 1980 & 2014 \\
\hline Marulanda & Marulanda & 5,267 & $-75,267$ & 1974 & 2015 \\
\hline Pensilvania & $\begin{array}{l}\text { San José } \\
\text { Pensilvania }\end{array}$ & 5,367 & $-75,133$ & 1974 & 2015 \\
\hline Pensilvania & Bolivia & 5,327 & $-75,117$ & 1964 & 2002 \\
\hline Pensilvania & Pensilvania & 5,386 & $-75,156$ & 1976 & 2014 \\
\hline Pensilvania & San Daniel & 5,378 & $-75,077$ & 1968 & 2002 \\
\hline Marmato & El Descanso & 5,500 & $-75,608$ & 1980 & 2013 \\
\hline Riosucio & Riosucio & 5,417 & $-75,717$ & 1970 & 2015 \\
\hline Riosucio & La Argentina & 5,467 & $-75,700$ & 1986 & 2014 \\
\hline Riosucio & La Manuelita & 5,367 & $-75,6833$ & 1970 & 2014 \\
\hline Supía & Rafael Escobar & 5,458 & $-75,641$ & 1971 & 2014 \\
\hline Anserma & Bellavista & 5,267 & $-75,800$ & 1963 & 2015 \\
\hline Chinchiná & $\begin{array}{l}\text { La Esperanza } \\
\text { Chinchiná }\end{array}$ & 4,636 & $-75,686$ & 1970 & 2014 \\
\hline Chinchiná & Cenicafé & 5,000 & $-75,600$ & 1951 & 2014 \\
\hline
\end{tabular}




\begin{tabular}{|c|c|c|c|c|c|}
\hline Chinchiná & La Francia & 4,969 & $-75,650$ & 1977 & 2014 \\
\hline Chinchiná & Naranjal & 4,972 & $-75,652$ & 1956 & 2014 \\
\hline Chinchiná & Ínsula & 5,002 & $-75,648$ & 1960 & 2012 \\
\hline Chinchiná & Montevideo & 4,996 & $-75,576$ & 1960 & 2012 \\
\hline Manizales & $\begin{array}{l}\text { Apto La } \\
\text { Nubia }\end{array}$ & 5,030 & $-75,467$ & 1968 & 2014 \\
\hline Manizales & Agronomía & 5,057 & $-75,495$ & 1956 & 2014 \\
\hline Manizales & Java & 5,014 & $-75,541$ & 1980 & 2014 \\
\hline Manizales & $\begin{array}{l}\text { Planta } \\
\text { Sancancio }\end{array}$ & 5,040 & $-75,502$ & 1960 & 2012 \\
\hline Manizales & $\begin{array}{l}\text { Subestación } \\
\text { Alta Suiza }\end{array}$ & 5,059 & $-75,485$ & 1975 & 2012 \\
\hline Manizales & $\begin{array}{l}\text { Subestación } \\
\text { Marmato }\end{array}$ & 5,066 & $-75,518$ & 1965 & 2010 \\
\hline Manizales & $\begin{array}{l}\text { Subestación } \\
\text { Uribe }\end{array}$ & 5,060 & $-75,542$ & 1975 & 2012 \\
\hline Neira & La Cristalina & 5,193 & $-75,433$ & 1970 & 2015 \\
\hline Neira & Neira & 5,267 & $-75,267$ & 1970 & 2015 \\
\hline Neira & $\begin{array}{l}\text { Neira Río } \\
\text { Tapias }\end{array}$ & 5,162 & $-75,607$ & 1975 & 2012 \\
\hline Palestina & $\begin{array}{l}\text { Apto } \\
\text { Santagueda }\end{array}$ & 5,083 & $-75,683$ & 1949 & 1989 \\
\hline Palestina & Arauca & 5,109 & $-75,705$ & 1962 & 2012 \\
\hline Palestina & El Recreo & 5,037 & $-75,647$ & 1970 & 2014 \\
\hline Palestina & Granja Luker & 5,067 & $-75,683$ & 1964 & 2014 \\
\hline Palestina & Santagueda & 5,073 & $-75,673$ & 1964 & 2014 \\
\hline Villamaría & Las Brisas & 4,902 & $-75,350$ & 1981 & 2014 \\
\hline Villamaría & Papayal & 4,951 & $-75,489$ & 1970 & 2014 \\
\hline Villamaría & $\begin{array}{l}\text { Montenegro } \\
\text { Molinos }\end{array}$ & 4,942 & $-75,469$ & 1963 & 2012 \\
\hline
\end{tabular}

TABLA III

ESTACIONES DE TEMPERATURA CON EVIDENCIA DE TENDENCIAS

\begin{tabular}{|c|c|c|c|c|c|}
\hline \multirow{2}{*}{$\begin{array}{l}\text { Municipio } \\
\text { Salamina }\end{array}$} & \multirow{2}{*}{$\begin{array}{c}\text { Estación } \\
\text { San Félix }\end{array}$} & \multirow{2}{*}{$\begin{array}{l}\text { Latitud } \\
5,3667\end{array}$} & \multirow{2}{*}{$\begin{array}{r}\text { Longitud } \\
-75,3667\end{array}$} & \multicolumn{2}{|c|}{$\begin{array}{c}\text { Periodo de } \\
\text { registro }\end{array}$} \\
\hline & & & & 1983 & 2015 \\
\hline Manzanares & Llanadas & 5,2 & $-75,1333$ & 1956 & 1998 \\
\hline Marquetalia & Santa Helena & 5,3175 & $-74,9961$ & 1981 & 2014 \\
\hline Supía & $\begin{array}{l}\text { Rafael } \\
\text { Escobar }\end{array}$ & 5,4578 & $-75,6411$ & 1982 & 2005 \\
\hline Anserma & Bellavista & 5,2667 & $-75,8$ & 1978 & 2015 \\
\hline Chinchiná & Cenicafé & 5 & $-75,6$ & 1951 & 2014 \\
\hline Chinchiná & Naranjal & 4,9719 & $-75,6522$ & 1956 & 2014 \\
\hline Manizales & Agronomía & 5,0567 & $-75,4953$ & 1956 & 2014 \\
\hline Palestina & Granja Luker & 5,0667 & $-75,6833$ & 1972 & 2014 \\
\hline Palestina & Santagueda & 5,0733 & $-75,6731$ & 1965 & 2014 \\
\hline
\end{tabular}

Finalizado el tratamiento con RClimdex de 55 series históricas de precipitación y 10 de temperatura para el departamento de Caldas, se identificó la existencia de tendencias crecientes y decrecientes, en aquellas estaciones que presentaron diferencias estadísticamente significativas $(\mathrm{P}$ Values $<0,10)$ en los indicadores climáticos.

Las estaciones con sus correspondientes años de registro y georreferenciación se exponen en las Tablas I y II. El análisis de tendencias arrojó diferencias estadísticamente significativas, en al menos un indicador de la variable precipitación, en 49 estaciones, las cuales se exponen en la Fig.1.

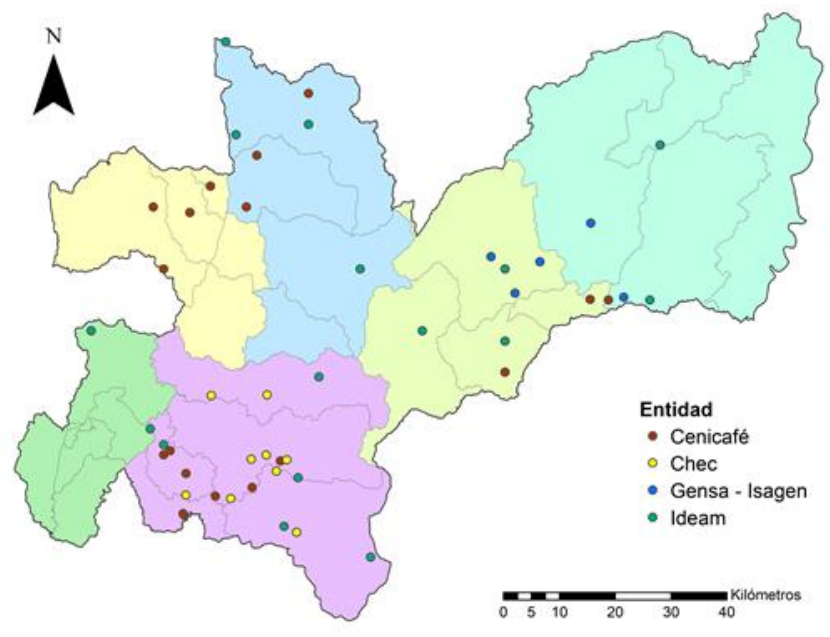

Fig. 1 Estaciones de precipitación con tendencias estadísticamente significativas.

El análisis con RClimdex estimó tendencias en 7 estaciones, en al menos un índice climático extremo relacionado a la variable temperatura; su localización se expone en la Fig.2.

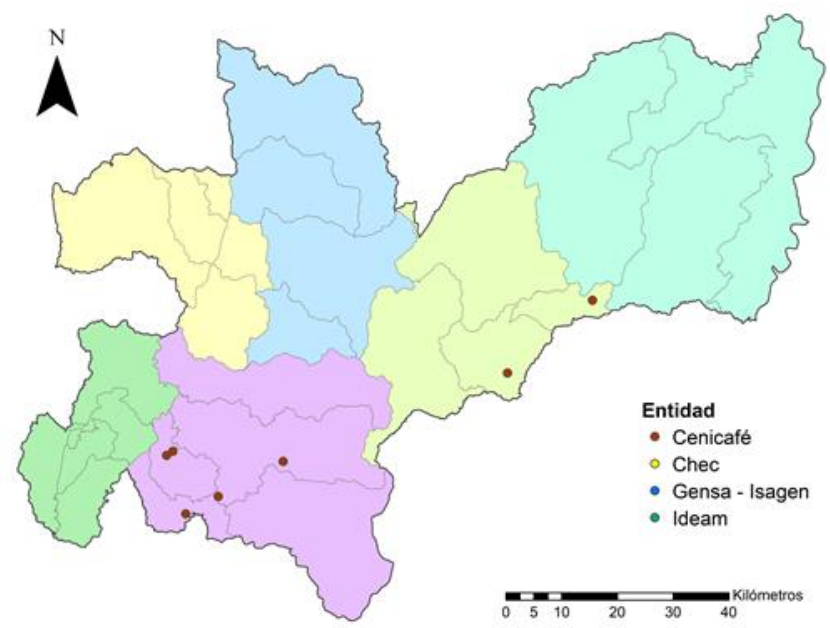

Fig. 2 Estaciones de temperatura con tendencias estadísticamente significativas.

\section{RESULTADOS}

El análisis de indicadores asociados a la variable precipitación se articula con la estimación de lluvias de diseño para obras de ingeniería. Por otra parte, los indicadores asociados a la variable temperatura se relacionan con los estudios y caracterizaciones de la climatología regional.

A continuación, se presentan en detalle los resultados de las tendencias identificadas en los índices climáticos extremos evaluados; cabe resaltar que el análisis se realizó en escala subregional.

\section{A. Cantidad máxima de precipitación en un día (RXIday)}

En el análisis del comportamiento histórico de la 
precipitación anual total y el número de días húmedos, se registraron tendencias en 22 estaciones localizadas en el departamento de Caldas, tal como se ilustra en la Fig.3.

Se registraron tendencias crecientes en las estaciones Guaymaral, Aguadas, La Palma, La Victoria, Pensilvania, San Daniel, Llanadas, Neira, Marmato, Planta Sancancio, El Recreo y Naranjal; mientras que, se reportaron tendencias decrecientes en las estaciones La Cascada, La Pastorita, Marulanda y Bellavista.

En las demás estaciones, no se registraron tendencias, crecientes o decrecientes, en las estaciones evaluadas, por lo cual se concluye que la variable tiene un comportamiento estable.

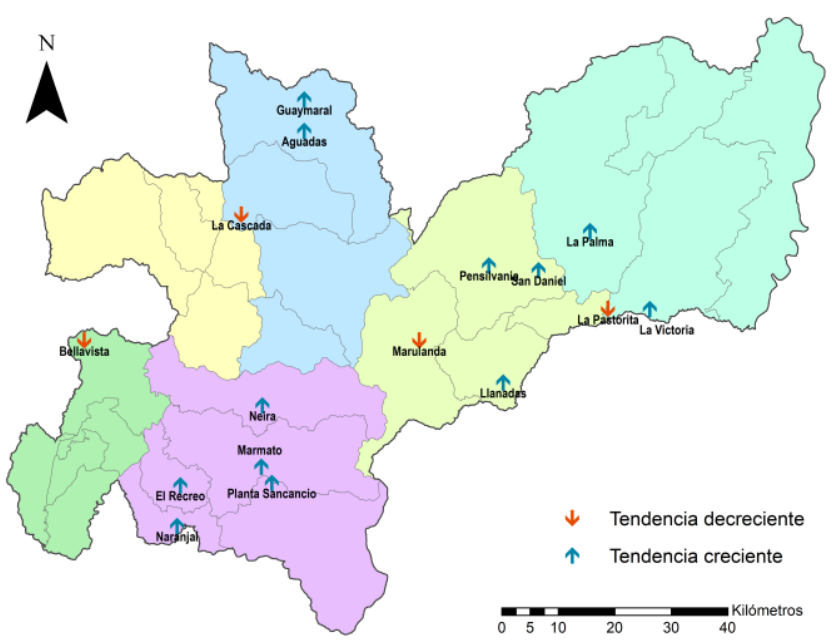

Fig. 3 Cantidad máxima de precipitación en un día (RX1day)

\section{B. Cantidad máxima de precipitación en 5 días (RX5day)}

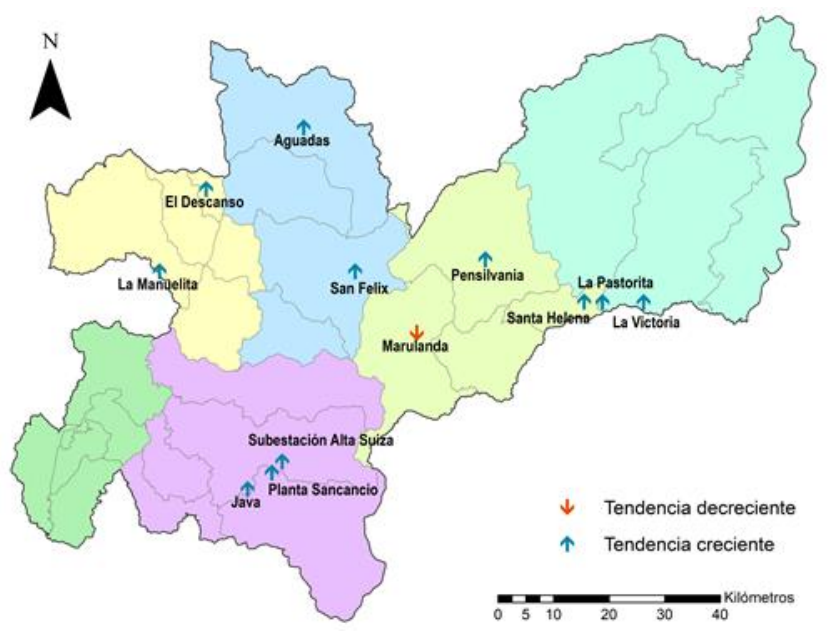

Fig. 4 Cantidad máxima de precipitación en 5 días (RX5day)

En el análisis del comportamiento histórico de la precipitación en un intervalo de 5 días, se registraron tendencias en 12 estaciones, como se ilustra en la Fig.4. Las estaciones Aguadas, San Félix, La Pastorita, La Victoria, Santa Helena, Pensilvania, El Descanso, La Manuelita, Subestación Alta Suiza, Planta Sancancio y Java mostraron tendencias crecientes, mientras que, la estación Marulanda, presentó tendencias decrecientes.

\section{C. Índice simple de intensidad diaria (SDII)}

En el análisis del comportamiento histórico de la precipitación anual total y el número de días húmedos, se registraron tendencias en 22 estaciones, tal como se ilustra en la Fig.5.

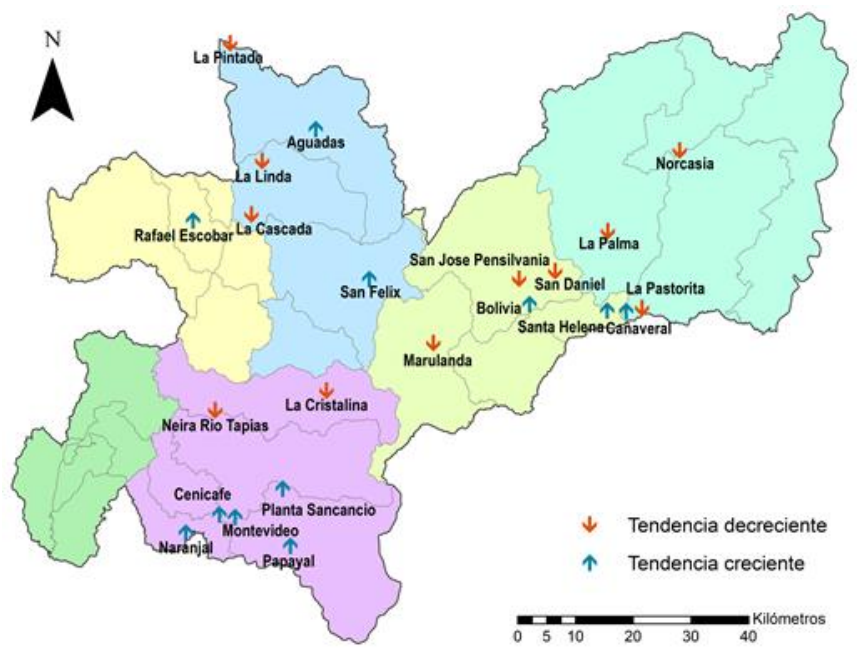

Fig. 5 Índice simple de intensidad diaria (SDII)

Tendencias crecientes fueron evidentes en las estaciones Aguadas, San Félix, Cañaveral, Santa Helena, Bolivia, Planta Sancancio, Montevideo, Papayal, Cenicafé, Montevideo, Naranjal y Rafael Escobar; por otro lado, se reportaron tendencias decrecientes en las estaciones La Pintada, La Linda, La Cascada, Norcasia, La Pastorita, La Palma, San Daniel, San José Pensilvania, Marulanda, La Cristalina y Neira Río Tapias.

\section{Días secos consecutivos (CDD)}

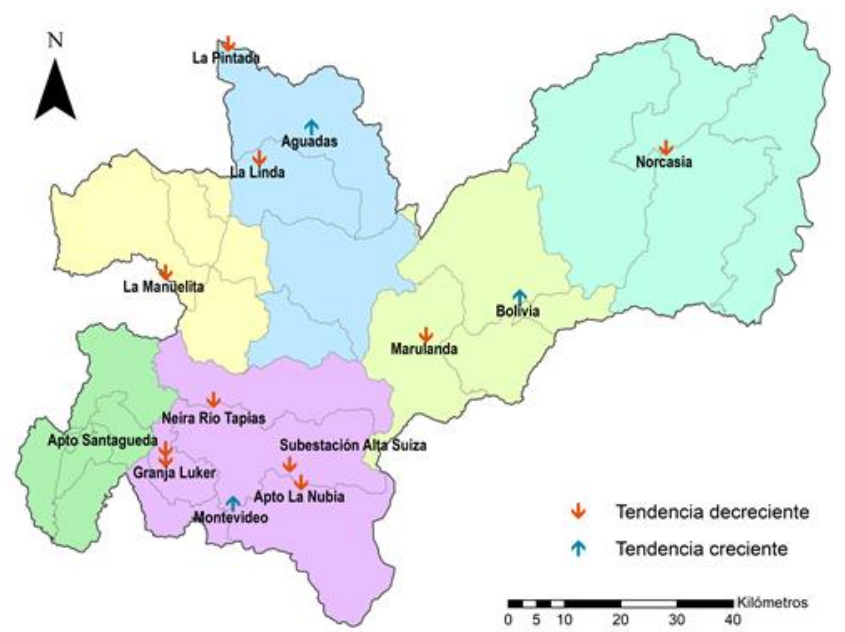

Fig. 6 Días secos consecutivos (CDD)

En el análisis del comportamiento histórico del número máximo de días secos consecutivos, se registraron tendencias en 13 estaciones, presentadas en la Fig.6.

Tendencias crecientes se manifestaron en las estaciones Aguadas, Bolivia y Montevideo y tendencias decrecientes en las estaciones La Pintada, La Linda, Norcasia, Marulanda, Subestación Alta Suiza, Apto La Nubia, Granja Luker, Apto 
Santagueda, Neira Río Tapias y La Manuelita.

\section{E. Días húmedos consecutivos (CWD)}

Según el análisis del comportamiento histórico del número máximo de días húmedos consecutivos, se registraron tendencias en 23 estaciones, como se ilustra en la Fig.7.

El análisis realizado presentó tendencias crecientes en las estaciones La Pintada, Norcasia, La Victoria, Cañaveral, La Pastorita, Pensilvania, San José Pensilvania, Manzanares, Papayal, Java, Ínsula, Neira Río Tapias, Bellavista, La Manuelita, Riosucio y La Argentina.

Se reportaron tendencias decrecientes en las estaciones Lanadas, Montenegro Molinos, Montevideo, Cenicafé y Arauca.

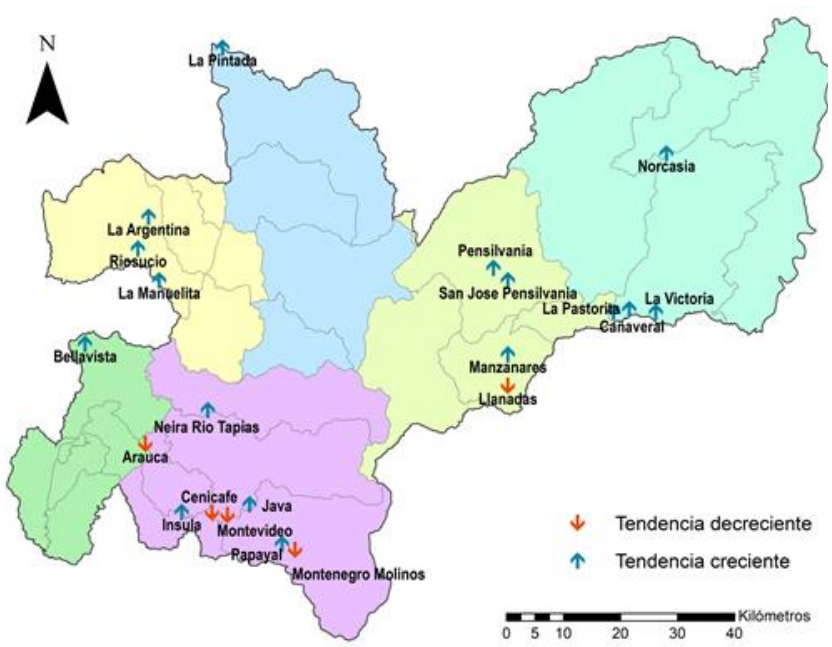

Fig. 7 Días húmedos consecutivos (CWD)

\section{F. Días muy húmedos(R95p)}

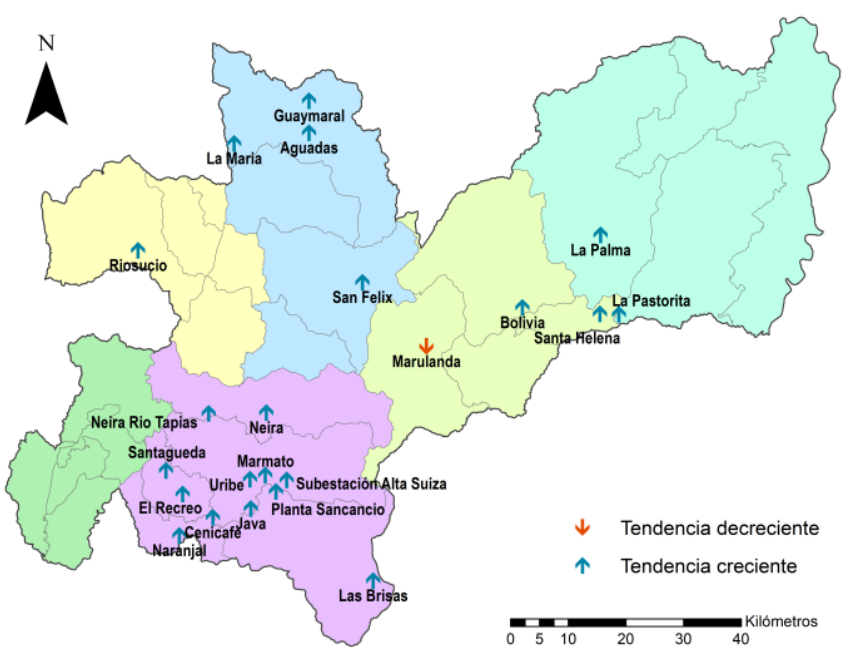

Fig. 8 Días muy húmedos (R95p)

El comportamiento histórico de la precipitación anual total que se encuentra por encima del percentil 95, registró tendencias en 22 estaciones, como se muestra en la Fig.8.

Se reportaron tendencias crecientes en las estaciones Guaymaral, Aguadas, La María, San Félix, La Palma, La Pastorita, Santa Helena, Bolivia, Neira, Marmato, Subestación Alta Suiza, Planta Sancancio, Java, Uribe, Cenicafé, Naranjal,
El Recreo, Santagueda, Neira Río Tapias y Riosucio, mientras que, solo se reportan tendencias decrecientes en la estación Marulanda.

\section{G. Días extremadamente secos ( $R 99 p)$}

En el análisis del comportamiento histórico de la precipitación anual total que se encuentra por encima del percentil 99, se registraron tendencias en 15 estaciones, como se ilustra en la Fig.9.

Tendencias crecientes fueron evidentes en las estaciones Aguadas, San Félix, Pensilvania, San Daniel, Bolivia, Santa Helena, Llanadas, Neira, Uribe, Subestación Alta Suiza, Planta Sancancio y Naranjal y tendencias decrecientes en las estaciones La Pintada, La Cascada, La Pastorita y Marulanda.

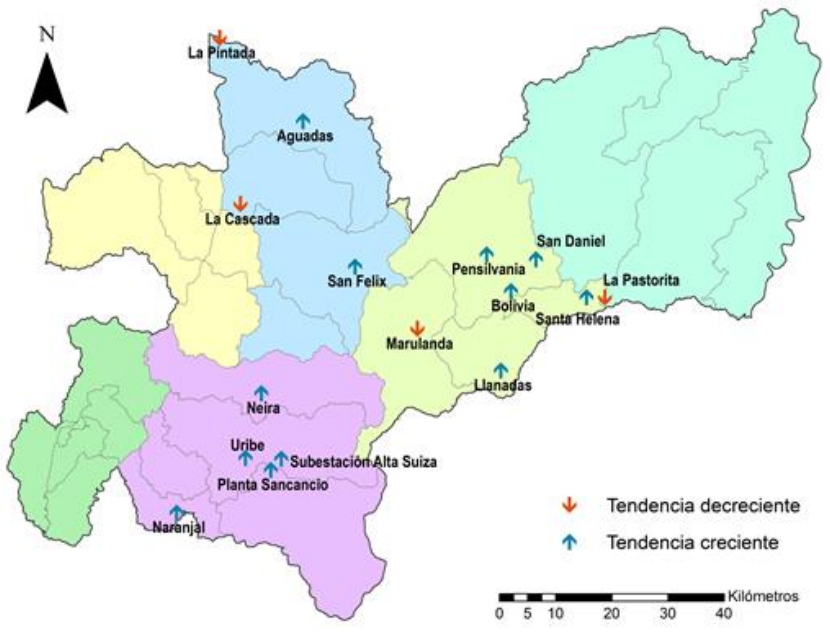

Fig. 9 Días extremadamente secos (R99p)

\section{H. Precipitación total anual en los días húmedos (PRCPTOT)}

El análisis del comportamiento histórico de la precipitación anual total en los días húmedos registró tendencias en 19 estaciones localizadas en el departamento de Caldas, tal como se ilustra en la Fig.10.

Se presentaron tendencias crecientes en las estaciones Aguadas, Norcasia, La Pastorita, Bolivia, Pensilvania, Subestación Alta Suiza, Planta Sancancio, Java, Naranjal, La Francia, Neira Río Tapias, Bellavista, La Manuelita, Riosucio, La Argentina y Rafael Escobar, mientras que, se reportaron tendencias decrecientes en las estaciones San Daniel, Montenegro Molinos y Montevideo. 


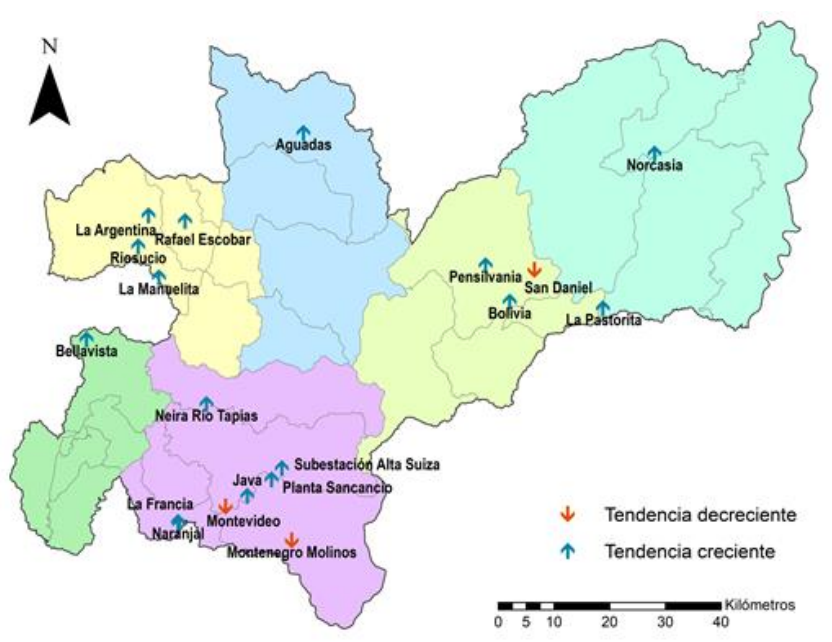

Fig. 10 Precipitación total anual en los días húmedos (PRCPTOT)

I. Valor mensual máximo de temperatura máxima diaria $(T X x)$

El comportamiento histórico de la máxima temperatura diaria registró tendencias en 3 estaciones, como se muestra en la Fig.11.

Los resultados del análisis en las estaciones Llanadas y Cenicafé mostraron tendencias crecientes; por su parte la estación Santa Helena presentó tendencias decrecientes.

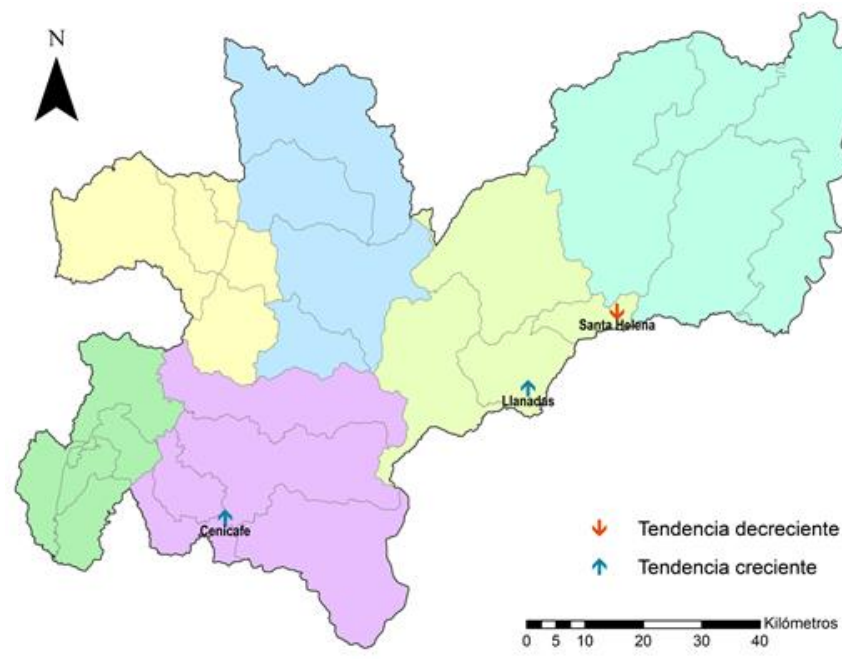

Fig. 11 Valor mensual máximo de temperatura máxima diaria (TXx)

\section{J. Valor mensual máximo de temperatura mínima diaria $(T N x)$}

En el análisis del comportamiento histórico de la mínima temperatura diaria, se registraron tendencias en 6 estaciones, como se ilustra en la Fig. 12.

Tendencias crecientes fueron expuestas en las estaciones Llanadas, Granja Luker, Agronomía, Santagueda, Cenicafé y Naranjal.

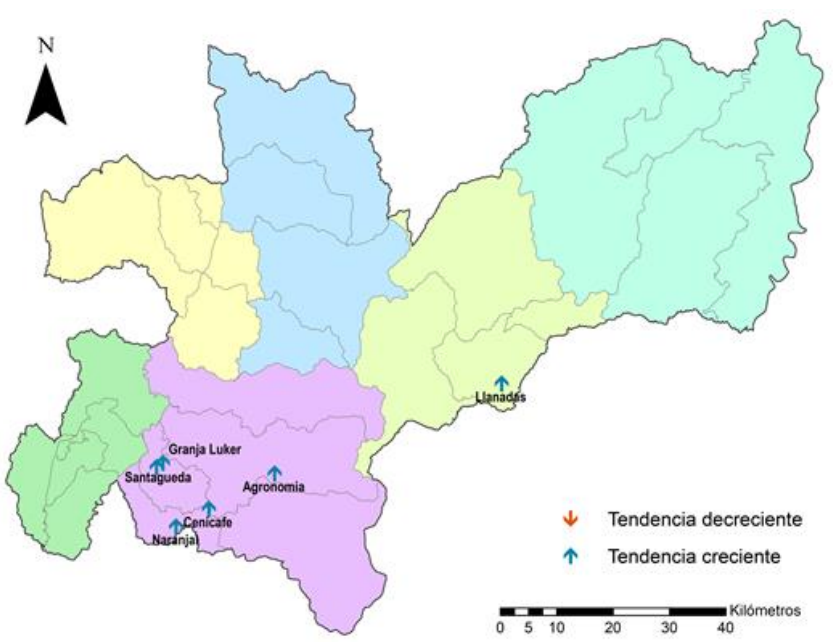

Fig. 12 Valor mensual máximo de temperatura mínima diaria (TNx)

$K$. Valor mensual mínimo de temperatura mínima diaria (TNn)

En el análisis del comportamiento histórico de la mínima temperatura diaria, se registraron tendencias en 7 estaciones, como se ilustra en la Fig.13.

En las estaciones Santa Helena, Llanadas, Agronomía, Granja Luker, Santágueda, Cenicafé y Naranjal se evidenciaron tendencias crecientes.

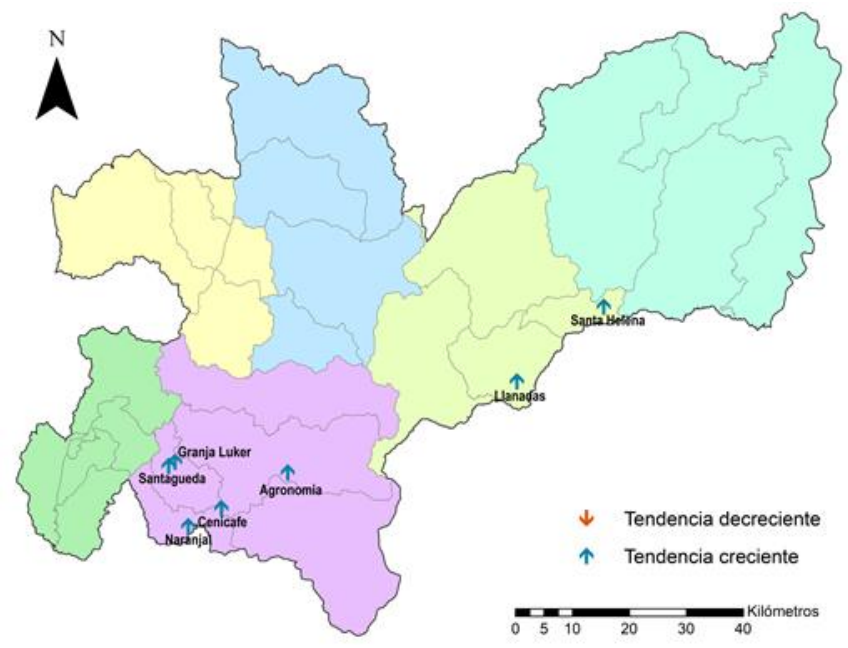

Fig. 13 Valor mensual mínimo de temperatura mínima diaria (TNn)

\section{CONCLUSIONES}

El análisis de tendencias de variables climatológicas considerando efectos de cambio climático permite asegurar con un nivel de seguridad del $90 \%$ que en el departamento podría presentarse un aumento en la cantidad precipitación y en la duración de los periodos húmedos. En la Tabla IV, se exponen los resultados más relevantes de los índices evaluados a nivel subregional, siendo $\mathrm{P}$ las tendencias positivas y $\mathrm{N}$ las tendencias negativas. 
TABLA IV

RESULTADOS DE ÍNDICES CLIMÁTICOS EXTREMOS

\begin{tabular}{lcccccc}
\hline \hline \multicolumn{1}{c}{ Índice } & Norte & $\begin{array}{c}\text { Magdalena } \\
\text { Caldense }\end{array}$ & $\begin{array}{c}\text { Alto } \\
\text { Oriente }\end{array}$ & $\begin{array}{c}\text { Alto } \\
\text { Occidente }\end{array}$ & $\begin{array}{c}\text { Occidente } \\
\text { Próspero }\end{array}$ & $\begin{array}{c}\text { Centro } \\
\text { Sur }\end{array}$ \\
\hline RX1day & $\mathrm{P}-\mathrm{N}$ & $\mathrm{P}-\mathrm{N}$ & $\mathrm{P}-\mathrm{N}$ & & $\mathrm{N}$ & $\mathrm{P}$ \\
RX5day & $\mathrm{P}$ & $\mathrm{P}$ & $\mathrm{P}-\mathrm{N}$ & $\mathrm{P}$ & & $\mathrm{P}$ \\
SDII & $\mathrm{P}-\mathrm{N}$ & $\mathrm{P}-\mathrm{N}$ & $\mathrm{P}-\mathrm{N}$ & $\mathrm{P}$ & & $\mathrm{P}-\mathrm{N}$ \\
CDD & $\mathrm{P}-\mathrm{N}$ & $\mathrm{N}$ & $\mathrm{P}-\mathrm{N}$ & $\mathrm{N}$ & & $\mathrm{P}-\mathrm{N}$ \\
CWD & $\mathrm{P}$ & $\mathrm{P}$ & $\mathrm{P}-\mathrm{N}$ & $\mathrm{P}$ & $\mathrm{P}$ & $\mathrm{P}-\mathrm{N}$ \\
R95p & $\mathrm{P}$ & $\mathrm{P}$ & $\mathrm{P}-\mathrm{N}$ & $\mathrm{P}$ & & $\mathrm{P}$ \\
R99p & $\mathrm{P}-\mathrm{N}$ & $\mathrm{N}$ & $\mathrm{P}-\mathrm{N}$ & & & $\mathrm{N}$ \\
PRCPTOT & $\mathrm{P}$ & $\mathrm{P}$ & $\mathrm{P}-\mathrm{N}$ & $\mathrm{P}$ & $\mathrm{P}$ & $\mathrm{P}-\mathrm{N}$ \\
TXx & & & $\mathrm{P}-\mathrm{N}$ & & & $\mathrm{P}$ \\
TNx & & & $\mathrm{P}$ & & & $\mathrm{P}$ \\
TNn & & & $\mathrm{P}$ & & & $\mathrm{P}$ \\
\hline
\end{tabular}

\section{REFERENCIAS}

[1] IPCC, 2014 "Climate change 2014: Synthesis Report. Contribution of Working Groups I, II and III to the Fifth Assessment Reporto of the Intergovernmental Panel on Climate Change." [Core Writing Team, R.K. Pachaurl and L.A. Meyer (eds)], IPCC, Geneva, Switzerland, $151 \mathrm{pp}$

[2] A. Velásquez y C. Rosales, "Los pasos de El Niño en Colombia 1980 - 2001." Universidad del Valle, Santiago de Cali, 2002.

[3] O. Ocampo, J. Vélez y A. Londoño, "Análisis de vulnerabilidad hídrica de la cuenca del río Chinchiná." Universidad Nacional de Colombia, Manizales, 2014.

[4] J. D. Pabón, "Búsqueda de Series de Referencia para el Seguimiento de la Señal Regional del Calentamiento Global." Cuadernos de Geografía, no 5, pp. 164-173, 1995.

[5] J. D. Pabón, "El cambio climático global y su manifestación en Colombia." Cuadernos de Geografía: Revista Colombiana de Geografía, no 12, pp. 111-119, 2003.

[6] J. D. Pabón, "Cambio climático en Colombia: tendencias en la segunda mitad del siglo XX y escenarios posibles para el siglo XXI" Rev. Acad. Colomb. Cienc., vol. 36, n ${ }^{\circ}$ 139, pp. 262-278, 2012.

[7] O. J. Mesa, G. Poveda y L. F. Carvajal, Introducción al clima de Colombia, Medellín: Universidad Nacional de Colombia, Sede Medellín, Facultad de Minas, Posgrado en Aprovechamiento de Recursos Hidráulicos, 1997.

[8] C. A. Pérez, G. Poveda, O. J. Mesa, L. Carvajal y A. Ochoa, "Evidencias de cambio climático en Colombia: Tendencias y cambios de fase y amplitud de los ciclos anual y semianual." Bulletin de l'Institute Francais d'Ètudes Andines, vol. 3, nº 27, pp. 537-546, 1998.

[9] R. Quintana Gómez, "Trends of Maximum and minimum Temperatures in Northern South America." Journal of Climate, vol. 6 , $\mathrm{n}^{\circ}$ 12, pp. 2104-2112, 1999. DOI: 10.1175/15200442(1999)012<2104:tomamt>2.0.co;2

[10] G. E. León, "Tendencia de la temperatura del aire en Colombia." Meteorología Colombiana, vol. 2, pp. 57-65, 2000.

[11] Pabón-Caicedo J.D., "Cambios en los patrones de temperatura media anual del aire y precipitación anual en los páramos de Colombia" de Páramos y Ecosistemas Alto Andinos de Colombia en Condiciones HotSpot \& Global Climatic Tensor, Bogotá D.C, Castaño-Uribe C, 2002, pp. 242-251.

[12] G. Poveda, J. I. Vélez, O. Mesa, A. Cuartas, J. Barco, R. Mantilla, J. F. Mejia, C. D. Hoyos, J. M. Ramírez, L. I. Ceballos, M. D. Zuluaga, P. A. Arias, B. A. Botero, M. I. Montoya, J. D. Giraldo y D. I. Quevedo, "Linking Long-Term Water Balances and Statistical Scaling to Estimate River Flows along the Drainage Network of Colombia." c Journal of Hydrologic Engineering, vol. 1, n 12, pp. 1-4, 2007. DOI: 10.1061/(ASCE)1084-0699(2007)12:1(4)

[13] L. Acevedo, "Estimación hidrológica bajo escenarios de cambio climático en Colombia.", Medellín: Tesis de Maestría, Universidad
Nacional de Colombia, 2009.

[14] G. Poveda, "Evidences of climate and environmental change on water resources and malaria in Colombia," de IOP Conf. Series, Earth Environ. Sci., 2009. DOI: 10.1088/1755-1307/6/9/292054

Instituto de Investigaciones Marinas y Costeras 'José Benito Vives De Andreis', "Capacity Building to Improve Adaptability to Sea Level Rise in Two Vulnerable Points of the Colombian Coastal Areas (Tumaco-Pacific Coast and Cartagena-Caribbean Coast) with Special Emphasis on Human Population under Poverty Conditions." INVEMAR, Santa Marta, 2008.

[16] IDEAM. Instituto Nacional de Hidrología Meteorología y Estudios Ambientales de Colombia, "Primera Comunicación Nacional de Colombia ante la Convención Marco de las Naciones Unidas sobre el Cambio Climático.” IDEAM, Bogotá D.C, 2001.

[17] IDEAM. Instituto Nacional de Hidrología Meteorología y Estudios Ambientales de Colombia., " $2^{\text {a }}$ Comunicación Nacional ante la Convención Marco de las Naciones Unidas sobre Cambio Climático.” IDEAM, Bogota D.C, 2010.

[18] IDEAM, "Atlas Climatológico de Colombia." Ministerio de Ambiente, Vivienda y Desarrollo Territorial. IDEAM., Bogotá D.C, 2005.

[19] IDEAM, "Atlas Climatológico de Colombia." 2015. [En línea]. Available: http://atlas.ideam.gov.co/.

[20] IDEAM, "Estudio Nacional del Agua.” IDEAM, Bogotá D.C, 2010.

[21] IDEAM, "Estudio Nacional del Agua 2014.” IDEAM, Bogotá D.C, 2015.

[22] G. Hurtado, "Análisis del comportamiento promedio y tendencias de largo plazo de la temperatura máxima media para las regiones hidroclimáticas de Colombia.” IDEAM, Bogotá D.C, 2012.

[23] G. Hurtado, "Análisis del comportamiento promedio y tendencias de largo plazo de las temperaturas mínimas medias para las regiones hidroclimáticas de Colombia.” IDEAM, Bogotá D.C, 2012.

[24] G. Hurtado, "Características y tendencias a largo plazo de las olas de calor y de frío en Colombia.” IDEAM, Bogotá D.C, 2012.

[25] R. Mayorga, G. Hurtado y H. Benavides, "Evidencias de cambio climático en Colombia con base en información estadística." Nota Técnica IDEAM, Bogotá D.C, 2011.

[26] E. P. Pérez, V. H. Ramírez y A. J. Peña, "Variabilidad espacial y temporal de la temperatura del aire en la zona cafetera colombiana." Investigaciones Geográficas, Boletín Del Instituto de Geografía, vol. 89, n 2016, p. 23-40, 2016. DOI: 10.14350/rig.38707

[27] H. O. Benavides y C. E. Rocha, "Indicadores que manifiestan cambios en el sistema climático de Colombia (Años y décadas más calientes y las más y menos lluviosas)." Nota Técnica IDEAM, Bogotá D.C, 2012.

[28] E. S. Rangel y J. E. Montealegre, “Análisis de las series del nivel del mar en el Pacífico colombiano y su relación con el cambio climático." Meteorología Colombiana, vol. 7, pp. 53-66, 2003. DOI: 10.13140/RG.2.1.2006.1206

[29] J. D. Pabón, "El cambio climático global y su manifestación en Colombia." Cuadernos de Geografía, Vols. \%1 de \%21-2, n 12, pp. 111-119, 2003.

[30] J. D. Pabón y J. A. Lozano, "Aspectos relacionados con las estimaciones globales y regionales del ascenso del nivel del mar y su aplicación a Colombia." Cuadernos de Geografía, n 14, pp. 97106, 2005

[31] Organización Meteorológica Mundial - OMM, "La información sobre el clima, un instrumento para reducir los riesgos de desastres." de Tercera Conferencia Mundial sobre el Clima, Ginebra, 2009.

[32] Organización Meteorológica Mundial - OMM, "El Clima y Tú." Ginebra, 2011.

[33] Organización Meteorológica Mundial - OMM, "El estado del clima mundial 2001-2010. Un decenio de fenómenos climáticos extremos." Ginebra, 2013.

[34] J. P. Marín, "Dinámica de los eventos hidroclimáticos extremos en la cuenca del río Chinchiná por efecto de variabilidad climática." Manizales: Universidad Nacional de Colombia - Sede Manizales, 
2017

[35] IPCC, "Gestión de los riesgos de fenómenos meteorológicos climático.” Ginebra, 2012.

[36] IPCC, "Cambio Climático 2007: Informe de Síntesis." Ginebra, 2007.

[37] J. Fallas y C. Valverde, "Inundaciones en Costa Rica y estudio de caso en una cuenca forestada del Caribe Costarricense: Evidencia de los últimos 34 años.” de Congreso Geoprocesamiento 2007.

[38] Programa de la Naciones Unidas para el Desarrollo - PNUD, "Panorama del Impacto ambiental de los recientes desastres naturales en América Latina y el Caribe.” 2000.

[39] Organización Meteorológica Mundial - OMM, "Resumen de conclusiones y cifras actualizadas sobre el cambio climático."

[40] J. Eslava, "Climatología y Diversidad Climática de Colombia." Rev. Acad. Colomb. Cienc, vol. 18, nº 71, pp. 507-538, 1993.

[41] R. Martínez, D. Ruiz, M. Andrade, L. Blacutt, D. Pabón, E. Jaimes, G. León, M. Villacís, J. Quintana, E. Montealegre y C. Euscátegui, "Sistemas del Clima de los Andes Tropicales." de Cambio climatico y Biodiversidad en los Andres Tropicales, Inter-American Institue for Global Change Research and Scientific Committe on Problems of the Environment, 2013, pp. 117-130.

[42] J. D. Pabón, J. A. Eslava, J. Pelkowski, G. J. Montoya y E. E. Vega, "Resultados de la simulación del clima por CCM3." Meteorología Colombiana, vol. 4, pp. 61-64, 2001.

[43] G. Poveda, "Mixed memory, (non) Hurst effect, and maximum entropy of rainfall in the tropical Andes." Advances in Water Resources, vol. 2, $\mathrm{n}^{\mathrm{o}}$ 34, pp. 243-256, 2011. DOI: 10.1016/j.advwatres.2010.11.007

[44] O. L. Ocampo, "Modelación hidrológica y agronómica de los efectos del cambio y la variabilidad climática en la producción cafetera de Caldas, Manizales." Universidad Nacional de Colombia - Sede Manizales, 2018.

[45] X. Zhang y F. Yang, RClimDex (1.0) Manual de Usuario, 2004. extremos y desastres para mejorar la adaptación al cambio Universidad Estatal a Distancia, San José, Costa Rica, 2007. Ginebra, 2011.

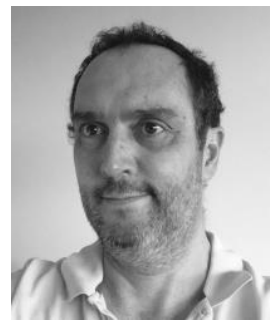

Jorge Julián Vélez Upegui Ingeniero Civil de la Facultad de Minas de la Universidad Nacional de Colombia Sede Medellín. Doctor En Planificación y Gestión de Recursos Hidráulicos de la Universidad Politécnica de Valencia, España.

Actualmente es Profesor Titular de la Universidad Nacional de Colombia Sede Manizales, y en esta universidad se ha desempeñado además de docente e investigador como Director del Laboratorio de Hidráulica, Director del Instituto de Estudios Ambientales IDEA y Director del Departamento de Ingeniería Civil. Sus investigaciones se han centrado en Procesos Hidrológicos de cuenca, Modelación hidrológica, Análisis espacio temporal de variables climáticas, Hidráulica fluvial, transporte y monitoreo de sedimentos en corrientes naturales, Riesgos por inundación y Diseño de redes de monitoreo ambiental.

ORCID: https://orcid.org/0000-0003-3856-1105

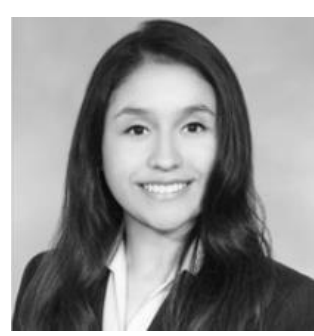

Tatiana Forero Hernández Ingeniera Civil, Especialista en Ingeniería Hidráulica y Ambiental. Estudiante de Maestría en Ingeniería- Recursos Hidráulicos de la Universidad Nacional de Colombia sede Manizales.

Vinculada al Grupo de Trabajo Académico en Ingeniería Hidráulica y Ambiental de la Universidad Nacional de Colombia Sede Manizales. Se desempeñó como contratista de la Universidad Autónoma de Manizales en los proyectos de Agendas de Cambio Climático y el Plan de Gestión Integral de Cambio Climático para el departamento de Caldas. Contratista del Instituto de Estudios Ambientales IDEA de la Universidad Nacional de Colombia Sede Manizales.

ORCID: https://orcid.org/0000-0002-8317-5177

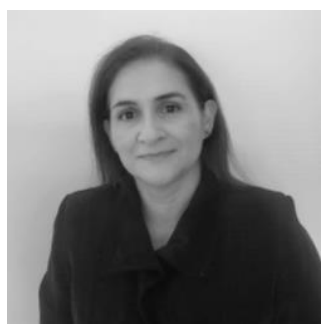

Olga Lucía Ocampo López es Ingeniera Química, Especialista en Ingeniería Ambiental, Maestría en Ingeniería Química, Doctora en Ingeniería Línea Automática de la Universidad Nacional de Colombia sede Manizales.

Actualmente es profesora Asociada de la Universidad Autónoma de Manizales e investigadora Senior de los grupos Diseño Mecánico y Desarrollo Industrial y Desarrollo Regional Sostenible.

Sus investigaciones se centran en cambio climático y variabilidad climática, modelación hidrológica, sostenibilidad, gestión ambiental, gestión de procesos y tecnología, responsabilidad social e innovación. Coordinó los proyectos de Agendas de Cambio Climático y el Plan de Gestión Integral de Cambio Climático para el departamento de Caldas.

ORCID: https://orcid.org/0000-0002-6394-977X

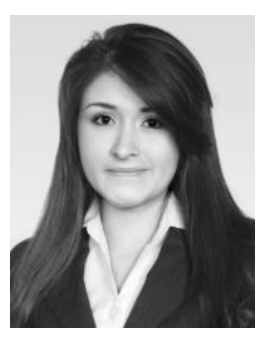

Jenny Paola Marín Salazar Ingeniera Civil, Especialista en Ingeniería Hidráulica y Ambiental. Maestría en Ingeniería- Recursos Hidráulicos de la Universidad Nacional de Colombia sede Manizales.

Ha sido contratista del Laboratorio de Hidráulica y del Instituto de Estudios Ambientales IDEA de la Universidad Nacional de Colombia Sede Manizales; se ha desempeñado como contratista del Instituto de Hidrología, Meteorología y de Estudios Ambientales de Colombia IDEAM. 